\title{
Avaliando o Uso das Ferramentas Educacionais no Ambiente Virtual de Aprendizagem Moodle
}

\author{
Marcelo A. Santana ${ }^{1}$, Baldoino S. Neto ${ }^{1}$, Evandro B. Costa ${ }^{1}$, Italo Carlo L. Silva ${ }^{1}$ \\ ${ }^{1}$ Instituto de Computação - Universidade Federal de Alagoas (UFAL) \\ Maceió- AL - Brasil
}

\begin{abstract}
Learning Management Systems (LMS) have contributed to the growth and popularity of distance learning modality. These LMSs are endowed with a variety of tools aimed at facilitating the process of teaching and learning. However, few studies have been conducted to evaluate the effectiveness of these tools. The objective of this study is to evaluate the use of these tools and their impact on the performance of students in the disciplines. This review was conducted in the LMS Moodle and the results has shown that most of the tools available in Moodle are being underutilized and that the relationship between them and student's performance is very low.
\end{abstract}

Resumo. Os ambientes virtuais de aprendizagem (AVA) têm contribuído para o crescimento e popularização da modalidade de ensino a distância. Tais AVAs são dotados de uma variedade de ferramentas cujo objetivo é facilitar o processo de ensino e aprendizagem. Entretanto, poucos estudos têm sido realizados para avaliar a eficácia destas ferramentas. O objetivo deste trabalho é avaliar o uso destas ferramentas e o impacto das mesmas sobre o desempenho dos estudantes nas disciplinas. Tal avaliação foi realizada no AVA Moodle e os resultados mostram que a maioria das ferramentas disponíveis no Moodle estão sendo subutilizadas e que a relação entre elas e o desempenho dos estudantes está muito baixa.

\section{Introdução}

O uso de ambientes virtuais de aprendizagem (AVA) tem sido um dos principais fatores que levaram ao rápido crescimento da educação a distância (EAD) [ABED 2014]. Um AVA permite que educadores compartilhem informações com alunos, produzam material de conteúdo, preparem trabalhos, testes, se envolvam em discussões, gerenciem classes à distância e permitam a aprendizagem colaborativa com fóruns, chats, armazenamento de arquivos, notícias, etc [Romero et al. 2008]. De acordo com [Magalhães et al. 2010], hoje em dia, um dos AVAs mais utilizados no mundo é o Moodle, que é uma plataforma de aprendizagem projetada para fornecer aos educadores, administradores e estudantes um sistema robusto, seguro e integrado para criar ambientes de aprendizagem personalizados [Moodle 2014]. Tem como característica manter registros detalhados de todas as atividades que os alunos realizam, gerando grandes volume de dados.

Técnicas de mineração de dados podem ser aplicadas para analisar grande volume de dados gerados em AVAs. Este procedimento é chamado EDM (Educational Data Mining). EDM está preocupado com o desenvolvimento de métodos para explorar os dados em ambientes educacionais e, através destes métodos, entender melhor os alunos e os contextos em que eles aprendem [Baker et al. 2010]. 
O Moodle também disponibiliza uma variedade de ferramentas que podem ser utilizadas por professores e estudantes para realizar diversas atividades de ensino e aprendizagem. Entretanto, existem poucos estudos que avaliem a importância destas ferramentas [Oeiras et al. 2006]. Diante disso, podemos levantar alguns questionamentos: As ferramentas disponíveis estão sendo utilizadas? Quais ferramentas de fato são úteis para o processo de ensino e aprendizagem dos estutantes? Quais as ferramentas mais utilizadas? As ferramentas estão influenciando no desempenho dos estudantes? As respostas a essas e outras indagações são o foco deste estudo. Para responder a essas questões foram utilizados dois métodos distintos.

Utilizamos como primeiro método a aplicação de técnicas de mineração de dados educacionais (EDM) sobre a base de dados do AVA Moodle, com o propósito de identificar quais são as ferramentas mais utilizadas e o impacto das mesmas sobre o desempenho dos estudantes nas disciplinas. Para isso foram aplicados algoritmos de classificação, para classificar os estudantes de acordo com suas notas e ferramentas usadas.

Devido a insuficiência de informações contidas na base de dados, tornou-se necessária a utilização de um método complementar capaz de extrair novos dados, que juntos aos dados extraídos no primeiro método permitiram responder os questionamentos citados anteriormente. Tal método consistiu na aplicação de um questionário voltado ao tutor online, a fim de identificar a sua disponibilidade de tempo, quais ferramentas disponibilizadas pelo AVA Moodle estão sendo efetivamente utilizadas e se existe alguma dificuldade no uso de tais ferramentas. Para a realização do estudo, foi indispensável o auxílio de uma ferramenta para extração, transformação e carga de dados e outra para aplicar algoritmos de mineração de dados. As ferramentas escolhidas foram Pentaho Data Integration [Pentaho 2014] e Weka [Weka 2014].

Os resultados apresentados comprovaram que a maioria das ferramentas disponíveis no Moodle estão sendo subutilizadas e que a relação entre elas não está tendo tanta influência no desempenho dos estudantes.

\section{Metodologia}

A fim de avaliar o uso e a influência das ferramentas de aprendizagem disponíveis no Moodle, aplicamos técnicas de EDM e um questionário para os tutores online, fundamentais no processo de EAD. Eles têm o papel mediar a comunicação de conteúdos entre o professor e os estudantes, acompanhar as atividades discentes e apoiar o professor da disciplina no desenvolvimento das atividades docentes [UFAL 2014].

A plataforma Moodle permite a transmissão e organização dos conteúdos com auxílio de ferramentas estáticas (páginas web, páginas de texto e conteúdo de pastas) e dinâmicas (chat, diário, fórum, glossário, quiz, wiki, livros, etc.). Este estudo foi realizado sobre as ferramentas dinâmicas, que foram escolhidas por possibilitarem uma maior interação entre os professores, tutores e estudantes. O estudo foi realizado seguindo as etapas descritas abaixo:

1. Pesquisa bibliográfica sobre Ambientes Virtuais de Aprendizagem, Mineração de Dados e Mineração de Dados na Educação (EDM);

2. Elaboração de questionário para os tutores online;

3. Seleção e tratamentos dos dados, objetivando preparar os dados para aplicação de técnicas de EMD; 
4. Realização dos experimentos;

5. Discussão dos resultados.

Todo procedimento foi realizado com o auxílio da ferramenta Pentaho Data Integration. Pentaho é um software de código aberto, desenvolvido em Java. A solução cobre as àreas de Extração, transformação e carga (ETL) dos dados, relatórios, OLAP e mineração de dados [Pentaho 2014], facilitando a criação de um modelo capaz de realizar os procedimentos de extração dos registros do Banco de Dados, seleção de atributos, discretização dos dados e até a geração do arquivo no formato compatível com o software de mineração. Para a execução dos algoritmos de DM utilizamos a ferramenta Weka, que é um software de código aberto que contém uma coleção de algoritmos de aprendizado de máquina para tarefas de mineração de dados [Weka 2014].

Desenvolvidas essas etapas, foi possível identificar a disponibilidade dos tutores online, as ferramentas mais utilizadas e a relação entre as ferramentas e o desempenho dos estudantes. Resultados e considerações sobre as etapas acima são apresentadas na seção 5 .

\section{Seleção e tratamento dos dados}

Para realização desse trabalho, foi utilizado um banco de dados real do AVA Moodle, cedido pela Universidade Federal de Alagoas (UFAL), contendo dez cursos de graduação à distância com cerca de 1800 alunos matriculados. Deste ambiente selecionou-se os quatros cursos que tinham o maior número de estudantes matriculados.

Seguindo o critério apresentado acima, foram selecionados os cursos de Sistema de Informação com 478 alunos matriculados; Física, com 211 alunos; Geografia, com 232; e Letras, com 179 alunos matriculados. Foi escolhido um subconjunto de atributos a fim de reduzir a dimensão e a complexidade do banco de dados. A escolha dos atributos se deu de acordo com a importância dos dados especificamente para o estudo das ferramentas e desempenho dos estudantes. Foram descartados atributos como dados cadastrais dos estudantes, dados cadastrais dos cursos e disciplinas entre outros que não iriam influenciar no estudo das ferramentas e desempenho dos estudantes. A tabela 1 apresenta os atributos selecionados.

Table 1. Atributos Selecionados

\begin{tabular}{|l|l|}
\hline \multicolumn{1}{|c|}{ Atributo } & \multicolumn{1}{c|}{ Descrição } \\
\hline Curso & Descrição do Curso \\
\hline AcessoTotal & Número total de acessos dos usuários \\
\hline MediaNotas & Média das notas dos estudantes por curso \\
\hline Assign & Quantidade de arquivos enviados pelo estudante \\
\hline Blog & Quantidade de acessos do estudante a ferramenta blog \\
\hline Book & Quantidade de acessos do estudante a ferramenta book \\
\hline Chat & Quantidade de acessos do estudante a ferramenta chat \\
\hline Choice & Quantidade de acessos do estudante a ferramenta choice \\
\hline Discussion & Quantidade de acessos do estudante a ferramenta discussion \\
\hline Forum & Quantidade de acessos do estudante a ferramenta forum \\
\hline Glossary & Quantidade de acessos do estudante a ferramenta glossary \\
\hline Message & Quantidade de acessos do estudante a ferramenta message \\
\hline Quis & Quantidade de acessos do estudante a ferramenta quiz \\
\hline Survey & Quantidade de acessos do estudante a ferramenta survey \\
\hline Wiki & Quantidade de acessos do estudante a ferramenta wiki \\
\hline Workshop & Quantidade de acessos do estudante a ferramenta workshop \\
\hline
\end{tabular}

Com objetivo de classificar os estudantes de acordo com as ferramentas utilizadas 
e as notas obtidas nas disciplinas, foram aplicados algoritmos de classificação. Alguns algoritmos de classificação conseguem lidar somente com atributos nominais. Para usá-los, os atributos numéricos devem primeiro ser "discretizados" [Witten et al. 2011]. Desse modo, para viabilizar a utilização desses algoritmos e também facilitar a interpretação dos resultados, os dados foram discretizados conforme os procedimentos abaixo.

O primeiro atributo a ser discretizado foi o atributo "AcessoTotal", onde foi dividido em três grupos de acordo com a média da quantidade total de acessos dos estudantes. Os valores que estavam acima da média foi atribuído o rótulo de "Alto", os que estavam próximo da média foram rotulados com "Medio" e por fim os valores que estavam abaixo da média ganharam o rótulo de "Baixo".

O atributo "MediaNota" também foi dividido em três grupos (A, B e C), a depender da média das notas obtidas pelos estudantes. A média das notas dos estudantes ficou por volta de 68. Os estudantes que tiveram notas maiores que a média ficaram no grupo "A" os que obtiveram as notas iguais ou próximas da média ficaram no grupo "B" e os que apresentaram notas abaixo da média ficaram no grupo "C".

Os atributos que representam a quantidade de acesso às ferramentas foram discretizados utilizando o mesmo método anterior, acrescentando somente o valor "sem acesso" quando a ferramenta em questão não tiver tido nenhum acesso dos estudantes. Vale destacar que existem diversas formas para o processo de discretização. Além disso, o número de classes e o número de instâncias em cada classe podem variar em função dos objetivos e das características particulares de cada estudo.

\section{Realizações dos experimentos}

\subsection{Objetivo}

O objetivo deste experimento é descobrir novos conhecimentos através das técnicas de EDM e aplicação de questionário. O experimento foi realizado como segue: (1) foram aplicadas técnicas de EDM sobre uma instância de uma base de dados do AVA Moodle, com o propósito de identificar quais as ferramentas mais usadas e o impacto das mesmas sobre o desempenho acadêmico dos estudantes nas disciplinas. O desempenho de cada estudante é definido como a média das notas obtidas nas disciplinas. Para isso foram aplicados algoritmos de classificação, para classificar os estudantes de acordo com suas notas e ferramentas usadas; (2) Foi aplicado um questionário aos tutores online a fim de identificar a disponibilidade de tempo dos tutores, quais ferramentas disponibilizadas pelo AVA Moodle estão sendo efetivamente utilizadas e se existem dificuldades no uso de tais ferramentas; e, por fim, (3) Foi realizado uma análise entre os resultados obtidos com os dois métodos a fim de responder todos os questionamentos da referida pesquisa.

\subsection{Aplicação e resultados dos algoritmos de mineração}

Após a extração, transformação e carga dos dados, o próximo passo foi definir qual técnica de mineração de dados seria aplicada. A título de pesquisa foi escolhida a técnica de classificação. A tarefa de classificação é uma forma de análise de dados que extrai um modelo que descreve as classes de dados importantes. Tais modelos, chamados de classificadores, prever categorizar os dados não classificados [Han et al. 2011]. Para identificar estas classes utilizam-se algoritmos, dentre os vários algoritmos existentes, o mais utilizado é o algoritmo de árvore de decisão. Para a realização do experimento foram 
utilizados 4 algoritmos de classificação: ID3, Random Forest, Simple Cart e J48. O algoritmo J48 obteve os melhores resultados. Ele é uma implementação open source Java do algoritmo C4.5 na ferramenta de mineração de dados Weka [Weka 2014]. O resultado dessa mineração pode ser visualizada na Figura 1.

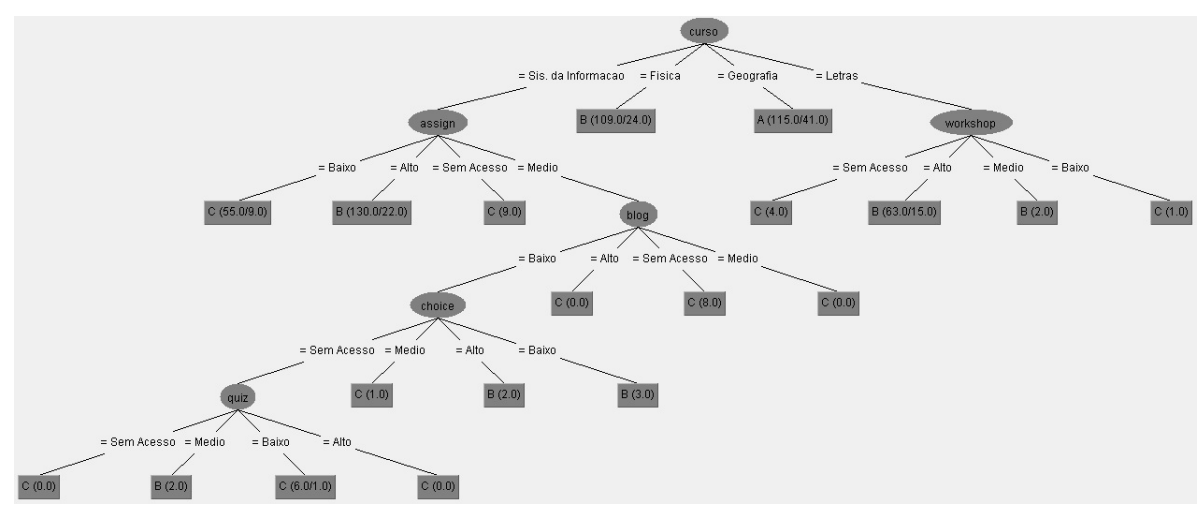

Figure 1. Resultado do algoritmo J48

A Figura 1 mostra o resultado do algoritmo $\mathrm{J} 48$ em forma de árvore, onde a árvore de decisão é a maneira gráfica de visualizar as consequências de decisões, onde cada nó interno (nó não-folha) denota um atributo, cada ramo representa um resultado, e cada nó folha (ou nó terminal) tem um rótulo de classe. O nó mais alto em uma árvore é o nó raiz [Han et al. 2011].

Analisando o resultado, é possível identificar como cada atributo se relaciona entre si para alcançar o atributo meta, que no experimento foi o atributo "MediaNota" que corresponde a média das notas dos estudantes discretizadas, (A,B,C), como mostrado na seção 3. Na Figura 1 é possível identificar que o curso de Geografia tem melhores médias "A" com 115 instâncias classificadas corretamente e 41 instâncias classificadas incorretamente. O curso de Física foi classificado tenho as médias das notas "B", os cursos de Sistemas de Informação e Letras a média das notas estão variando de acordo com o uso das ferramentas disponíveis no AVA Moodle. Coincidentemente, os estudantes dos cursos que obtiveram as melhores médias das notas fazem parte dos cursos que menos utilizam as ferramentas disponíveis no AVA.

Outro resultado analisado foi o da estatística Kappa, onde o resultado foi 0.5922 , ou seja, considerado moderado de acordo com a tabela de [Landis e Koch. 1977]. Essa estatística é de grande importância, pois permite avaliar o nível de concordância e ligação dos dados, sendo que, se o número ficar próximo de 0 (zero) significa uma maior discordância das informações, e ficando o mais próximo de 1 (um) indica uma maior ligação e concordância dos dados [Simões and Paulo 2011].

Outro ponto importante são os índices de revocação (recall) e precisão (precision), o resultado da revocação (recall) gerada pelo experimento foi de 0.769 , onde esse valor é representado pelo número de documentos relevantes recuperados pelo sistema dividido pelo numero total de documentos relevantes contidos no sistema [Witten et al. 2011]. A precisão (precision) gerada pelo experimento foi de 0.778 , seu valor é obtido pelo número de documentos recuperados que são relevantes dividido pelo número total de documentos recuperados na busca [Witten et al. 2011]. 
Entre os cursos escolhidos, também foi possível identificar as ferramentas mais utilizadas pelos estudantes de cada curso. Como mostra a Figura 2, entre todas as ferramentas disponíveis a ferramenta fórum e envio e recebimento de arquivo (assign) foram as mais usadas.

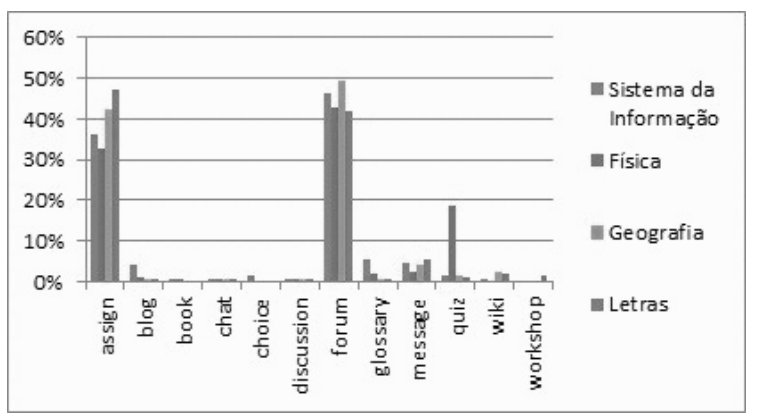

Figure 2. Gráfico das Ferramentas mais usadas

\subsection{Aplicação e resultados do questionário}

O questionário foi aplicado em dezessete (17) tutores online, que atuam nos diversos cursos de graduação a distância da Universidade Federal de Alagoas (UFAL). Foi escolhido aplicar o questionário aos tutores online, pois eles são fundamentais no processo de EAD. Eles têm o papel de mediar a comunicação entre os professores e estudantes, acompanhar as atividades discentes, conforme o cronograma do curso e apoiar o professor da disciplina no desenvolvimento das atividades docentes [UFAL 2014]. O questionário apresentou dez (10) questões, que foram divididas em três partes. A primeira envolvendo perguntas relacionadas à disponibilidade de tempo dos tutores, a segunda teve como objetivo identificar o uso das ferramentas disponibilizadas pelo AVA Moodle e a terceira parte tem a finalidade de apontar o uso de outras ferramentas fora do AVA Moodle. A tabela 2 apresenta as perguntas do questionário.

A partir dos resultados do questionário foi possível chegar as seguintes conclusões.

- Com as primeiras perguntas foi possível identificar que não há preocupação com relação ao tempo exigido para as realizações das tarefas direcionadas aos tutores. Considerando que o prazo exigido ao tutor online para responder aos estudantes é de 48 hrs. Como podemos verificar na Figura 3.

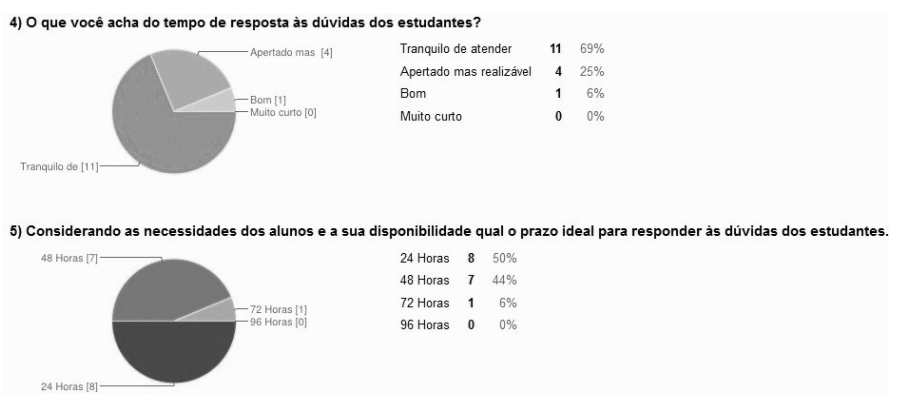

Figure 3. Gráfico relacionado ao tempo dos tutores 
Table 2. Perguntas do questionário

\begin{tabular}{|c|c|}
\hline Enunciado das perguntas & Alternativas \\
\hline Há quanto tempo você é tutor? & $\begin{array}{l}\text { Primeiro semestre } \\
\text { De dois a três semestre } \\
\text { Mais de três semestre }\end{array}$ \\
\hline De quantas disciplinas você é tutor ao mesmo tempo? & $\begin{array}{l}\text { Uma única disciplina } \\
\text { Duas } \\
\text { Três } \\
\text { Mais de três } \\
\end{array}$ \\
\hline Onde geralmente você atende às demandas da tutoria? & $\begin{array}{l}\text { Faculdade/Escola } \\
\text { Trabalho } \\
\text { Casa } \\
\text { Outros }\end{array}$ \\
\hline O que você acha do tempo de resposta às dúvidas dos estudantes? & $\begin{array}{l}\text { Tranquilo de atender } \\
\text { Apertado mas realizável } \\
\text { Bom } \\
\text { Muito curto }\end{array}$ \\
\hline $\begin{array}{l}\text { Considerando as necessidades dos alunos e a sua disponibilidade qual o prazo ideal para } \\
\text { responder às dúvidas dos estudantes. }\end{array}$ & $\begin{array}{l}24 \text { Horas } \\
48 \text { Horas } \\
72 \text { Horas } \\
96 \text { Horas } \\
\end{array}$ \\
\hline $\begin{array}{l}\text { Qual a frequência de uso das ferramentas?(Blog, Book, Chat, Choice, Discussion, Forum, } \\
\text { Glossary, Message, Quiz, Survey, Wiki, Workshop) }\end{array}$ & $\begin{array}{l}\text { Nunca Usei } \\
\text { Já usei } \\
\text { Uso sempre em algumas disciplinas } \\
\text { Uso Sempre }\end{array}$ \\
\hline $\begin{array}{l}\text { Marque o nível de dificuldade encontrado por você em cada uma das ferramentas.(Blog, Book, } \\
\text { Chat, Choice, Discussion, Forum, Glossary, Message, Quiz, Survey, Wiki, Workshop) }\end{array}$ & $\begin{array}{l}\text { Nunca Usei } \\
\text { Muito difícil } \\
\text { Razoável } \\
\text { Muito Fácil }\end{array}$ \\
\hline Aponte as dificuldades relatadas pelos estudantes nas ferramentas. & $\begin{array}{l}\text { Nunca utilizaram } \\
\text { Encontra dificuldade com frequência } \\
\text { Alguns encontram } \\
\text { Não encontram dificuldades }\end{array}$ \\
\hline Você utiliza alguma outra ferramenta fora do ambiente Moodle? Quais? & Questão aberta \\
\hline Na sua opinião o que você acha que poderia melhor no ensino a distância da UFAL? & Questão aberta \\
\hline
\end{tabular}

- A segunda parte foi para identificar quais ferramentas os tutores mais utilizam e quais dificuldades encontradas no seu uso. De acordo com o questionário a ferramenta mais utilizada é o fórum com $81 \%$ das respostas afirmando que sempre usa a ferramenta. Apesar de não encontrarem nenhum tipo de dificuldade no uso de outras ferramentas. Como pode-se notar nas Figuras 4 e 5.

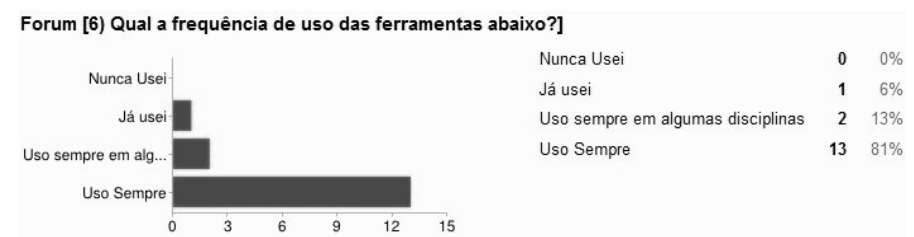

Figure 4. Uso da ferramenta fórum

- Por fim, foi possível apontar que apesar dos vários ferramentas disponível no AVA Moodle os tutores ainda utilizam outras ferramentas fora do AVA Moodle. O mais citado foi o uso do e-mail como forma de comunicação entre os tutores, professores e estudantes.

Para mais detalhes o resultado completo do pesquisa está disponível no site http://www.resultsearch.xpg.com.br/.

\section{Discussão}

Dos resultados obtidos com a aplicação dos experimentos, concluiu-se que há pouco uso das ferrametas disponíveis no AVA Moodle, e que as ferramentas de envio e recebimento de arquivos (assign) e o fórum são as mais usadas, e a relação entre elas e o desempenho dos estudantes dos estudantes está muito baixa. 


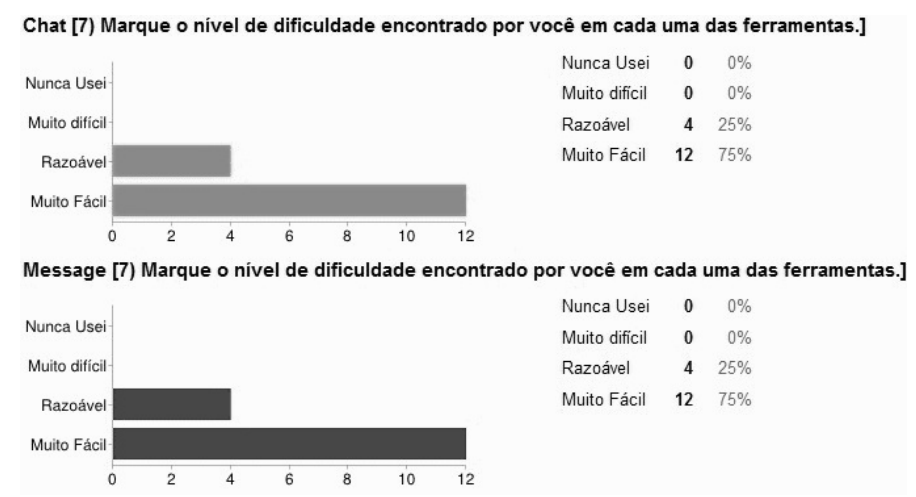

Figure 5. Dificuldade encontrada nas ferramentas

O principal aspecto a ser destacado da seção 4.2, é mostrar que não apresentam indícios de influência no desempenho dos estudantes pelo uso das ferramentas. Podemos notar isso observando os dados do curso de Geografia e Física que mesmo obtendo as melhores médias de notas não tem relação com o uso das ferramentas. Também nessa seção foi possível mostrar que a maioria das ferramentas não estão sendo usadas.

Na seção 4.3, é importante destacar que os tutores online realmente não usam ou não conhecem a maioria das ferramentas, mesmo à considerando de fácil uso. Vale a pena destacar que a maioria dos tutores ( $75 \%$ dos entrevistados) tem experiência, exercendo a função de tutor online a mais de três (3) semestres.

Os resultados apresentados nesse estudo atingiram o seu objetivo, evidenciando que a utilização das ferramentas no AVA Moodle estão sendo subutilizadas e que essas ferramentas não estão tendo tanta influência no desempenho dos estudantes. Vale a pena destacar que o estudo foi realizado somente sobre a base de dados do AVA Moodle da Universidade Federal de Alagoas, podendo obter resultados diferentes utilizando outras bases de dados.

\section{Trabalhos relacionados}

[Romero and Ventura 2010] em seus trabalhos têm fornecido referências valiosas em relação à descoberta de conhecimento em ambientes virtuais de aprendizagem. Em um dos seus estudos eles desenvolvem um tutorial para aplicação de técnica de mineração de dados no sistema AVA Moodle, com o objetivo de apresentar teoria e prática a todos os interessados nesta nova área de pesquisa, em especial para professores on-line e os administradores de e-learning. Mostrando todo o processo passo a passo para a mineração de dados no Ambiente Virtual de Aprendizagem, bem como a forma de aplicar as principais técnicas de mineração de dados utilizados.

[Gottardo et al. 2012] utilizam técnicas de mineração de dados educacionais com algoritmos de classificação tendo como objetivo de gerar inferências sobre o desempenho dos estudantes a partir de dados coletados em séries temporais, conseguindo uma taxa de precisão de próxima a 75\%, em etapas iniciais da realização do curso.

A pesquisa de [Azeredo et al. 2012] faz um estudo no sentido de analisar indicadores de relevância nas postagens dos fóruns de discursão. Utilizando técnicas de mineração de dados, onde foi desenvolvido o software MineraFórum. Os resultados 
apresentados pelo MineraFórum foram comparados com um questionário aplicado com docentes, onde se concluiu que a média das análises das postagens, calculada pelo MineraFórum, é semelhante à média das avaliações dos professores.

[Kumar et al. 2011] faz um estudo focado no AVA Moodle, onde os autores tem como objetivo descobrir a escolha do melhor e mais adequado sistemas de e-learning fazendo uma comparação com outros ambientes virtuais de aprendizagem. Chegando a conclusão que a plataforma moodle em comparação com outros sistemas de e- learning é um sistema ideal para educação à distância.

Já [Mozzaquatro and Medina 2008] fazem uma avaliação das características funcionais e não funcionais do sistema moodle através de questionário e observação. Para o estudo foi realizada a análise e avaliação da estrutura e organização do AVA Moodle, utilizado em duas Instituições de Ensino Superior. Dentre várias conclusões obtidas com o estudo, vale destacar a conclusão que se referem às ferramentas onde, foi possível concluir que as formas de interação no ambiente foram consideradas boas e a ferramenta de interação que mais contribuiu para a aprendizagem foi o fórum, seguido da ferramenta de livros e questionários.

Em resumo, diversos trabalhos investigam a aplicação de questionários ou técnicas de mineração de dados educacional com o objetivo de identificar padrões nos AVAs existentes. Entretanto, de forma geral, são estudos que focam apenas uma única ferramenta ou utilizam apenas dados de uma única fonte. Neste estudo, em contribuição aos que foram apresentados nesta seção, propõe realizar o estudo focando não apenas uma ferramenta e utilizando dois métodos distintos a fim de conseguir mais informações relevantes.

\section{Conclusão e trabalhos futuros}

Os resultados obtidos nesse estudo apontam a viabilidade de realizar inferências relativas ao uso das ferramentas disponíveis no AVA Moodle. Estas inferências podem ser úteis para professores no sentido de ajudá-los no desenvolvimento de conteúdo e no processo de ensino e aprendizagem, aos tutores na intenção de auxiliá-los no processo de avaliação e participação dos estudantes e aos alunos tornando mais motivados e presentes nos cursos à distância.

Como continuidade deste estudo, alguns pontos pendentes ainda deverão vir a ser considerados para a melhoria da pesquisa realizada, como os que se seguem: realizar o estudo com base de dados de outras universidades; inserir novos algoritmos de classificação; aumentar a quantidade de amostras, inserir novos atributos e melhorar o processo de discretização dos dados; desenvolver um software capaz de auxiliar aos professores e tutores na escolha das ferramentas de acordo com os perfis dos cursos ou perfis dos estudantes.

\section{References}

ABED (2014). Associação brasileira de educação a distância. http: / / www . abed . org.br/site/pt/. Acesso em junho 2014.

Azeredo, B. F. T., Behar, P. A., and Reategui, E. B. (2012). Indicadores de relevância para análise de fóruns de discussão. Simpósio Brasileiro de Informática na Educação, Rio de Janeiro. 
Baker, R. et al. (2010). Data mining for education. International encyclopedia of education, 7:112-118.

Gottardo, E., Kaestner, C., and Noronha, R. V. (2012). Previsão de desempenho de estudantes em cursos ead utilizando mineração de dados: uma estratégia baseada em séries temporais. Simpósio Brasileiro de Informática na Educação, Rio de Janeiro.

Han, J., Kamber, M., and Pei, J. (2011). Data Mining: Concepts and Techniques. Morgan Kaufmann Publishers Inc., San Francisco, CA, USA, 3rd edition.

Kumar, S., Gankotiya, A., and Dutta, K. (2011). A comparative study of moodle with other e-learning systems. In Electronics Computer Technology (ICECT), 2011 3rd International Conference on, volume 5, pages 414-418.

Magalhães, E., Gomes, V., Rodrigues, A., Santos, L., and Conte, T. (2010). Impacto da usabilidade na educação a distância: Um estudo de caso no moodle ifam. In Proceedings of the IX Symposium on Human Factors in Computing Systems, IHC '10, pages 231-236, Porto Alegre, Brazil, Brazil. Brazilian Computer Society.

Moodle (2014). Moodle - modular object-oriented dynamic learning environment. https://moodle.org/. Acesso em maio 2014.

Mozzaquatro, M. P. and Medina, D. R. (2008). Avaliação do ambiente virtual de aprendizagem moodle sob diferentes visões: aspectos a considerar. Revista Novas Tecnologias na Educação.

Oeiras, J. Y. Y., Freire, F. M. P., Lachi, R. L., and da Rocha, H. V. (2006). Design de ferramentas de comunicação baseado em gêneros de discurso. In Proceedings of VII Brazilian Symposium on Human Factors in Computing Systems, IHC '06, pages 56-65, New York, NY, USA. ACM.

Pentaho (2014). Pentaho - pentaho data integration. http: / / www . pentaho. com/. Acesso em junho 2014.

Romero, C. and Ventura, S. (2010). Educational data mining: A review of the state of the art. Systems, Man, and Cybernetics, Part C: Applications and Reviews, IEEE Transactions on, 40(6):601-618.

Romero, C., Ventura, S., and García, E. (2008). Data mining in course management systems: Moodle case study and tutorial. Computers \& Education, 51(1):368-384.

Simões, P. and Paulo, R. (2011). Utilização de técnicas de aprendizado de máquina no reconhecimento de entidades nomeadas no português. Revista letrônicas do Centro Universitário e-Xacta, Belo Horizonte.

UFAL (2014). Universidade federal de alagoas. http://www.ufal.edu.br/ cied/nucleo-de-tutoria/estrutura-do-nucleo. Acesso em junho 2014.

Weka (2014). Weka - the university of waikato. http://www.cs.waikato.ac. $\mathrm{nz} / \mathrm{ml} /$ weka/. Acesso em junho 2014.

Witten, I. H., Frank, E., and Hall, M. A. (2011). Data Mining: Practical Machine Learning Tools and Techniques. Morgan Kaufmann Publishers Inc., San Francisco, CA, USA, 3rd edition. 